\title{
21. A Note on (E.R.)integral and Fourier Series
}

\author{
By Teruo IKEgami
}

(Comm. by K. Kunugr, M.J.A., Feb. 12, 1960)

1. Introduction and notations. In this note we shall consider (E.R.)integral of rather special type." ${ }^{1)}$ Let $\varepsilon$ be the set of all step functions defined on the finite closed interval $[a, b]$. In $\mathcal{E}$ we shall introduce a topology and a rank so that $\mathcal{E}$ becomes a ranked space. Let $F$ be the closed set in $[a, b], \nu$ be an integer and $f$ be a function in $\mathcal{E}$, then we shall define a neighbourhood of $f, V(F, \nu ; f)$ as the set of all functions $g \in \mathcal{E}$ such that there exist in $\mathcal{E}$ functions $p(x)$ and $r(x): g(x)-f(x)=p(x)+r(x)$ which satisfy the following conditions:

[1] $r(x)$ is zero for all $x$ in $F$,

[2] we have $\int_{\alpha}^{b}|p(x)| d x<2^{-\nu}$,

$\left[3_{1}\right]$ for every $c, d: a \leqq c<d \leqq b$ we have $\left|\int_{c}^{d} r(x) d x\right|<2^{-\nu} \cdot{ }^{1)}$

A neighbourhood $V(F, \nu ; f)$ is called of rank $\nu$, if we have mes $\{[a, b]$ $-F\}<2^{-\nu}$. A sequence of neighbourhoods $\left\{u_{n}\right\}=\left\{V\left(F_{n}, \nu_{n} ; f_{n}\right)\right\}$ is called fundamental sequence, if we have

(1) $u_{0} \supseteq u_{1} \supseteq \cdots \supseteq u_{n} \supseteq \cdots$, the rank of $u_{n}$ is $\nu_{n}$,

(2) $\nu_{0} \leqq \nu_{1} \leqq \cdots \leqq \nu_{n} \leqq \cdots$,

(3) $f_{2 n}=f_{2 n+1}, \nu_{2 n}<\nu_{2 n+1}, n=0,1,2, \cdots$

Further we shall add the conditions:

$\left(1^{*}\right)$ the sequence $\left\{u_{n}\right\}$ has the property $P$, that is, there exists a function $\phi(n)(n=0,1,2, \cdots)$ such that $\phi(n)>0$ for $n=0,1,2, \cdots$, $\lim _{n \rightarrow \infty} \phi(n)=0$, and for every measurable set $E$ contained in $[a, b]$ whose measure is less than mes $\left\{[a, b]-F_{n}\right\}$, we have

$$
\int_{E}\left|f_{n}(x)\right| d x \leqq \phi(n)
$$

1) The investigation of (E.R.)integral originates from the note of Prof. K. Kunugi: Application de la méthode des espaces rangés à la théorie de l'intégration. I, Proc. Japan Acad., 32, 215-220 (1956). In original note the condition [3 $3_{1}$ is weaker than the present one, that is,

[3] we have $\left|\int_{a}^{b} r(x) d x\right|<2^{-\nu}$.

While the condition $\left[3_{1}\right]$ was first considered by Dr. S. Nakanishi: Sur la dérivation de l'intégrale (E.R.)indéfinie. I, Proc. Japan Acad., 34, 199-204 (1958), which makes the indefinite integral continuous.

In this present note we owe also the note of Prof. K. Kunugi: Sur une généralisation de l'intégrale, Fundamental and Applied Aspects of Mathematics, 1-30, Research Institute of Applied Electricity, Hokkaido University. 
$\left(2^{*}\right)$ there exists an integer $\kappa(\kappa \geqq 2)$ which satisfies for every $n$ the inequality:

$$
\kappa \cdot \operatorname{mes}\left\{[a, b]-F_{n+1}\right\} \geqq \operatorname{mes}\left\{[a, b]-F_{n}\right\} .
$$

In the following, we shall consider mainly the fundamental sequences which satisfy the conditions $\left(1^{*}\right),\left(2^{*}\right){ }^{2}{ }^{2}$ For such sequence the limits

$$
\lim _{n \rightarrow \infty} f_{n}(x), \quad \lim _{n \rightarrow \infty} \int_{c}^{a} f_{n}(x) d x
$$

exist (the former limit exists except the set of measure zero and the latter exists for all $c, d$ ) and the limit of integrals depends only on the limit function. This function is called (E.R.)integrable and this fundamental sequence is called a defining sequence of this function. Thus we can define for $a \leqq x \leqq b$

$$
F(x)=\lim _{n \rightarrow \infty} \int_{\alpha}^{x} f_{n}(t) d t
$$

this is expressed by (E.R.) $\int_{\alpha}^{x} f(x) d x$, where $f(x)=\lim _{n \rightarrow \infty} f_{n}(x)$. It is known that $F(x)$ is continuous. ${ }^{3)}$

2. Next we shall enter one of the main results of this note. Before proving the theorem we describe auxiliary articles.

Lemma. If $f(x)$ is (E.R.)integrable in $[a, b]$ and Lebesgue integrable in $[c, d](a \leqq c<d \leqq b)$, then there exists fundamental sequence $\left\{V\left(F_{n}, \nu_{n} ; f_{n}\right)\right\}$ and we have

$$
\begin{gathered}
\lim _{n \rightarrow \infty} f_{n}(x)=f(x) \quad \text { for almost all } x, \\
\lim _{n \rightarrow \infty} \int_{c}^{a}\left|f_{n}(x)-f(x)\right| d x=0 .
\end{gathered}
$$

In the following we employ ourselves in the study on Fejér kernel and Poisson kernel, nevertheless we state the theorem for kernels of more abstract character. In this apparently infructuous generalization we intend to make the type of proof clear and not to repeat the details of proof for other kernels, for example, kernels $K_{n}^{r}(x), 0<r<1$. $^{\text {) }}$

We shall consider functions $K_{n}(x)(n=1,2, \cdots)$ satisfying the following conditions and call them kernels.

$\left.1^{\circ}\right) K_{n}(x)$ is an absolutely continuous periodic function with period $2 \pi$ and $K_{n}(-x)=K_{n}(x)$.

$2^{\circ}$ ) for every $x_{0}$ we have $\int_{-\pi}^{\pi} K_{n}\left(x-x_{0}\right) d x=2 \pi$.

$\left.3^{\circ}\right) \quad \int_{-\pi}^{\pi}\left|K_{n}(x)\right| d x \leqq C$, where $C$ denotes a constant independent of $n$.

2) Unless otherwise stated, we shall use the terminology "fundamental sequence" in this sense.

3) Cf. S. Nakanishi: Loc. cit. p. 201. (1935).

4) For this kernel, cf. A. Zygmund: Trigonometrical Series, 42, 48, Warsaw 
$\left.4^{\circ}\right)$ for every $\lambda>0$ we have $\lim _{n \rightarrow \infty} A_{n}(\lambda)=0$, where $A_{n}(\lambda)=\operatorname{Max}\left|K_{n}(x)\right|$ for $\lambda \leqq x \leqq \pi$.

$5^{\circ}$ ) for every $\lambda>0$ there exists $M(\lambda)$ and we have for all $n, B_{n}(\lambda)$ $\leqq M(\lambda)$, where $B_{n}(\lambda)$ denotes the absolute variation of $K_{n}(x)$ on $[\lambda, \pi]$.

By $2^{\circ}$ ), $3^{\circ}$ ), $4^{\circ}$ ) our kernels are quasi-positive.

Theorem 1. Let $f(x)$ be (E.R.)integrable on $[-\pi, \pi]$ and at $x_{0}$ there exist finite limits $f\left(x_{0}-\right), f\left(x_{0}+\right)$, then for every $n$ the function $f(x) \cdot K_{n}(x)$ is also (E.R.)integrable and we have

$$
\lim _{n \rightarrow \infty} 1 / 2 \pi(E . R .) \int_{-\pi}^{\pi} f(x) K_{n}\left(x-x_{0}\right) d x=\left[f\left(x_{0}+\right)+f\left(x_{0}-\right)\right] / 2 .
$$

Proof. Without loss of generality, we may suppose that $x_{0}=0$, and $f(x)$ is continuous at $x=0$, for otherwise we may define anew $f(x)=[f(x)+f(-x)] / 2$ and $f(0)=[f(0+)+f(0-)] / 2 ;\{f(x)-f(0)\} K_{n}(x)$ is (E.R.)integrable ${ }^{5)}$ and there exists a fundamental sequence $\left\{V\left(F_{k}\right.\right.$, $\left.\left.\nu_{k} ; \varphi_{k}\right)\right\}$ satisfying the following conditions

$$
\lim _{k \rightarrow \infty} \varphi_{k}(x)=f(x)-f(0) \quad \text { for almost all } x,
$$

$$
\begin{gathered}
\text { (E.R.) } \int_{-\pi}^{\pi}\{f(x)-f(0)\} K_{n}(x) d x=\lim _{k \rightarrow \infty} \int_{-\pi}^{\pi} \varphi_{k}(x) K_{n}(x) d x,{ }^{6)} \\
\lim _{k \rightarrow \infty} \int_{-\lambda}^{\lambda}\left|\{f(x)-f(0)\}-\varphi_{k}(x)\right| d x=0,
\end{gathered}
$$

where $\lambda$ is chosen as follows: by continuity of $f(x)$, for arbitrary $\varepsilon>0$ there exists $\lambda>0$ such that $|x|<\lambda$ implies $|f(x)-f(0)|<\varepsilon$.

First of all, we shall show that the limit

$$
\lim _{k \rightarrow \infty} \int_{o}^{d} \varphi_{k}(x) K_{n}(x) d x
$$

exists for every $c, d$ and for all $n$. In fact for $k^{\prime}>k$ we get $\mid \int_{c}^{d} \varphi_{k^{\prime}}(x)$ - $K_{n}(x) d x-\int_{o}^{d} \varphi_{k}(x) K_{n}(x) d x|=| \int_{o}^{a}\left\{p_{k^{\prime}, k}(x)+r_{k^{\prime}, k}(x)\right\} K_{n}(x) d x \mid,(*)$; where $r_{k^{\prime} k}$ vanishes on the set $F_{k}, \int_{-\pi}^{\pi}\left|p_{k^{\prime}, k}(x)\right| d x<2^{-\nu} k$ and for every $\alpha<\beta$ we have $\left|\int_{\alpha}^{\beta} r_{k^{\prime}, k}(x) d x\right|<2^{-\nu_{k}}$. Therefore let $R_{k^{\prime}, k}(x)$ be an indefinite integral of $r_{k^{\prime}, k}$, then $\left|R_{k^{\prime}, k}(x)\right|<2^{-\nu}$ for all $x$. Integrating by parts, the last integral is evaluated as follows: $\quad(*) \leqq \int_{-\pi}^{\pi}\left|p_{k^{\prime}, k}(x)\right| \cdot\left|K_{n}(x)\right| d x$ $+\left|R_{k^{\prime}, k}(d)\right| \cdot\left|K_{n}(d)\right|+\left|R_{k^{\prime}, k}(c)\right| \cdot\left|K_{n}(c)\right|+\int_{c}^{a}\left|R_{k^{\prime}, k}(x)\right| \cdot\left|K_{n}^{\prime}(x)\right| d x \leqq 2^{-\nu_{k}}\left(3 M_{n}\right.$
$\left.+W_{n}\right) \rightarrow 0 \quad(k \rightarrow \infty)$,

5) Cf. S. Nakanishi: L'intégrale (E.R.) et la théorie des distributions, Proc. Japan Acad., 34, 565-570, Proposition 1 (1958).

6) S. Nakanishi: Loc. cit. Lemme 1. 
where $M_{n}=\operatorname{Max}\left|K_{n}(x)\right|$ for $-\pi \leqq x \leqq \pi, W_{n}=\int_{-\pi}^{\pi}\left|K_{n}^{\prime}(x)\right| d x$.

Hence we can write $\lim _{k \rightarrow \infty} \int_{-\pi}^{\pi} \varphi_{k}(x) K_{n}(x) d x=\lim _{k \rightarrow \infty} \int_{-\pi}^{-\lambda}+\lim _{k \rightarrow \infty} \int_{-\lambda}^{\lambda}+\lim _{k \rightarrow \infty} \int_{\lambda}^{\pi}$

$=\lim _{k \rightarrow \infty}\left(I_{k, n}^{(1)}+I_{k, n}^{(2)}+I_{k, n}^{(3)}\right) . \quad$ By $\quad(2.2)\left|\int_{-\lambda}^{\lambda}\left[\{f(x)-f(0)\}-\varphi_{k}(x)\right] K_{n}(x) d x\right|$

$\leqq \operatorname{Max}_{|x| \leqq \lambda}\left|K_{n}(x)\right| \cdot \int_{-\lambda}^{\lambda}\left|\{f(x)-f(0)\}-\varphi_{k}(x)\right| d x \rightarrow 0 \quad(k \rightarrow \infty)$.

Therefore $\lim _{k \rightarrow \infty}\left|I_{k, n}^{(2)}\right|=\lim _{k \rightarrow \infty}\left|\int_{-\lambda}^{\lambda} \varphi_{k}(x) K_{n}(x) d x\right|=\left|\int_{-\lambda}^{\lambda}\{f(x)-f(0)\} K_{n}(x) d x\right|$

$\leqq \varepsilon \cdot \int_{-\pi}^{\pi}\left|K_{n}(x)\right| d x \leqq C \cdot \varepsilon\left(\right.$ by $\left.\left.3^{\circ}\right)\right)$. Consequently

$$
\lim _{k \rightarrow \infty}\left|I_{k, n}^{(2)}\right| \leqq C \cdot \varepsilon, \quad n=1,2,3, \cdots .
$$

Next we shall evaluate $I_{k, n}^{(3)}$. Let $k_{0}$ be an integer such that $M(\lambda) \cdot 2^{-k_{0}}<\varepsilon$, and let $J_{0}=\int_{-\pi}^{\pi}\left|\varphi_{k_{0}}(x)\right| d x$. Then we select an integer $n_{0}$ so that $n \geqq n_{0}$ implies $A_{n}(\lambda)\left(3 \cdot 2^{-k_{0}}+J_{0}\right)<\varepsilon$. Let $n \geqq n_{0}$ and $k>k_{0}$. $I_{k, n}^{(3)}=\int_{\lambda}^{\pi} \varphi_{k}(x) K_{n}(x) d x=\int_{\lambda}^{\pi}\left\{\varphi_{k}(x)-\varphi_{k_{0}}(x)\right\} K_{n}(x) d x+\int_{\lambda}^{\pi} \varphi_{k_{0}}(x) K_{n}(x) d x$ $=\int_{\lambda}^{\pi}\left\{p_{k, k_{0}}(x)+r_{k, k_{0}}(x)\right\} K_{n}(x) d x+\int_{\lambda}^{\pi} \varphi_{k_{0}}(x) K_{n}(x) d x . \quad p_{k, k_{0}}(x), r_{k, k_{0}}(x)$ satisfy the same conditions as described above. Using again integration by parts we shall obtain the inequality $\left|I_{k, n}^{(3)}\right| \leqq 3 \cdot 2^{-\nu} k_{0} \cdot A_{n}(\lambda)+2^{-\nu} k_{0} B_{n}(\lambda)$ $+A_{n}(\lambda) \cdot J_{0} \leqq \varepsilon+\varepsilon=2 \varepsilon$. Therefore, for $n \geqq n_{0}$ we get $\lim _{k \rightarrow \infty}\left|I_{k, n}^{(3)}\right| \leqq 2 \varepsilon$. Quite similarly we shall obtain $\lim _{k \rightarrow \infty}\left|I_{k, n}^{(1)}\right| \leqq 2 \varepsilon$ for $n \geqq n_{0}$. Collecting the results, we conclude that $\mid$ (E.R.) $\int_{-\pi}^{\pi}\{f(x)-f(0)\} K_{n}(x) d x \mid \leqq(4+C) \varepsilon$, for $n \geqq n_{0}$. This inequality and $2^{\circ}$ ) give the proof of Theorem 1 .

3. Let $f(x)$ be (E.R.)integrable in $[-\pi, \pi]$, then for every $n$ the functions $f(x) \cos n x, f(x) \sin n x$ are also (E.R.)integrable in $[-\pi, \pi]$. Accordingly we shall obtain the Fourier series

$$
1 / 2 \cdot a_{0}+\sum_{n=1}^{\infty}\left(a_{n} \cos n x+b_{n} \sin n x\right),
$$

where Fourier coefficients $a_{n}, b_{n}$ are given by the following (E.R.)integrals

$$
\pi a_{n}=\text { (E.R.) } \int_{-\pi}^{\pi} f(x) \cos n x d x, \quad \pi b_{n}=\text { (E.R.) } \int_{-\pi}^{\pi} f(x) \sin n x d x .
$$

Now we shall consider summability of (3.1). In Theorem 1, if we take Fejér kernels $K_{n}^{r}(x)(r \geqq 1)$ and Poisson kernel we shall obtain immediately that under the hypothesis of Theorem 1, the series (3.1) is summable $(C, r)$ and summable $(A)$ at the point $x_{0}$ to the value $\left[f\left(x_{0}+\right)+f\left(x_{0}-\right)\right] / 2$. 
Further we shall consider summability $(C, r), 0<r<1$.

Theorem 2. Let $f(x)$ be (E.R.)integrable in $[-\pi, \pi]$. If $f(x)$ is bounded in a neighbourhood of the point $x_{0}$, then for the $(C, r)$ means of (3.1) $\sigma_{n}^{r}(x)$ we obtain the relation $\sigma_{n}^{r}\left(x_{0}\right)=o\left(n^{1-r}\right)$, where $0<r<1$.

In the definition of fundamental sequence $\left(1^{*}\right)$ we shall add the condition:

$$
\sum_{k=0}^{n} \phi(k)=o\left(n^{r}\right) \quad \text { for some } r, \quad 0<r<1,
$$

and in the next theorem (and for this theorem only) we shall say that the function $f(x)$ is (E.R.)integrable if there exists a fundamental sequence defining $f(x)$ and satisfies (3.2).

Theorem 3. Let $0<r<1$. Under the same conditions as Theorem 1, the series (3.1) is $(C, r)$ summable at the point $x_{0}$ to the value $\left[f\left(x_{0}+\right)+f\left(x_{0}-\right)\right] / 2$.

Proof. Without loss of generality we may suppose that $x_{0}=0$, $f\left(x_{0}\right)=0$ and $f(x)$ is continuous at $x_{0}$. Let $\left\{V\left(F_{k}, \nu_{k} ; f_{k}\right)\right\}$ be a fundamental sequence defining $f(x)$. Using the same notation as in Theorem 1 , we get $\pi \sigma_{n}^{r}(0)=\lim _{k \rightarrow \infty}\left(I_{k, n}^{(1)}+I_{k, n}^{(2)}+I_{k, n}^{(3)}\right)$ and (2.3) is valid here also. If we show that $\lim _{n \rightarrow \infty} I_{n, n}^{(3)}=0, \lim _{n \rightarrow \infty}\left(\lim _{k \rightarrow \infty} I_{k, n}^{(3)}-I_{n, n}^{(3)}\right)=0$ the theorem will be proved. First, $\left|I_{n, n}^{(3)}\right|=\left|\int_{\lambda}^{\pi}\left\{f_{n}(x)-f_{v}(x)\right\} K_{n}^{r}(x) d x+\int_{\lambda}^{\pi} f_{0}(x) K_{n}^{r}(x) d x\right|$ $\leqq\left|\sum_{k=0}^{n-1} \int_{\lambda}^{\pi} p_{k}(x) K_{n}^{r}(x) d x+\sum_{k=0}^{n-1} \int_{\lambda}^{\pi} r_{k}(x) K_{n}^{r}(x) d x\right|+\left|\int_{\lambda}^{\pi} f_{0}(x) K_{n}^{r}(x) d x\right|$ $\leqq A_{n}^{r}(\lambda)\left\{\sum_{k=0}^{n-1} \int_{-\pi}^{\pi}\left(\left|p_{k}(x)\right|+\left|r_{k}(x)\right|\right) d x+\int_{-\pi}^{\pi}\left|f_{0}(x)\right| d x\right\}$. By definition, $r_{k}(x)$ $=f_{k+1}(x)-f_{k}(x)-p_{k}(x)$ vanishes for $x$ in $F_{k}$ and inequality (1.1) leads to the result $\int_{\lambda}^{\pi}\left|r_{k}(x)\right| d x \leqq \int_{[\lambda, \pi]-F_{k}}\left\{\left|f_{k+1}(x)\right|+\left|f_{k}(x)\right|+\left|p_{k}(x)\right|\right\} d x \leqq \kappa \cdot \phi(k+1)$ $+\phi(k)+2^{-\nu_{k}}$. Therefore $\left|I_{n, n}^{(3)}\right| \leqq A_{n}^{r}(\lambda)\left\{\int_{-\pi}^{\pi}\left|f_{0}(x)\right| d x+2 \cdot \sum_{k=0}^{n-1} 2^{-\nu_{k}}+(\kappa+1)\right.$ $\left.\cdot \sum_{k=0}^{n} \phi(k)\right\} \rightarrow 0 \quad(n \rightarrow \infty) .^{7} \quad$ Secondly, for sufficiently large $n$ and for $k>n$ we have as before $\left|I_{k, n}^{(3)}-I_{n, n}^{(3)}\right|=\left|\int_{\lambda}^{\pi}\left\{f_{k}(x)-f_{n}(x)\right\} K_{n}^{r}(x) d x\right|=\mid \int_{\lambda}^{\pi} p_{k, n}(x) K_{n}^{r}(x)$ $\cdot d x+\int_{\lambda}^{\pi} r_{k, n}(x) K_{n}^{r}(x) d x \mid \leqq 3 A_{n}^{r}(\lambda) \cdot 2^{-\nu_{n}}+2^{-{ }^{-}} C^{\prime} n^{1-r} r^{-1} \lambda^{-r}$. But, as $\nu_{n} \geqq[n / 2]$ we have $\lim _{n \rightarrow \infty} 2^{-\nu n} n^{1-r}=0$. Therefore $\lim _{n \rightarrow \infty}\left(\lim _{k \rightarrow \infty} I_{k, n}^{(3)}-I_{n, n}^{(3)}\right)=0$.

As an immediate corollary of this theorem we shall refer to the result which is regarded, if it is allowed to say so, to show the advantage of our integral. ${ }^{8}$

7) It is known that $K_{n}^{r}(t)=O\left(n^{-r} t^{-r-1}\right)$ for $n t \geqq 1$.

8) In the case of Denjoy-Fourier series, it is known that this circumstance can not occur. Cf. S. Pollard: The summation of Denjoy-Fourier series, Proc. London Math. Soc., 27, 216 (1928). Cf. also, A. Zygmund: Loc. cit. p. 268. 
Corollary. Let $f(x)$ be (E.R.) integrable in $[-\pi, \pi]$ in the sense of Theorem 3. If there is a set $E$ of which measure is positive and at each point $x$ of $E$ the limits $f(x+), f(x-)$ exist and finite, then for Fourier coefficients of (3.1) we have

$$
a_{n}=o\left(n^{r}\right), \quad b_{n}=o\left(n^{r}\right) .
$$

At the end of this section, we shall state a theorem without proof.

Theorem 4. Let $f(x)$ be (E.R.)integrable in $[-\pi, \pi]$. If we set for $0 \leqq r<1,0 \leqq x<2 \pi$,

$$
f(r, x)=1 / 2 \pi(E . R .) \int_{-\pi}^{\pi} f(t)\left(1-r^{2}\right) /\left\{1-2 r \cos (t-x)+r^{2}\right\} d t,
$$

then the function $f(r, x)$ is harmonic in the unit circle, and if further the indefinite integral of $f(x), F(x)=(E . R.) \int_{-\pi}^{x} f(t) d t$ is derivable at $x_{0}$, then $f(r, x)$ has the limit $F^{\prime}\left(x_{0}\right)$ when $(r, x) \rightarrow\left(1, x_{0}\right)$ along any path not touching the circle.

4. Remark. So far we have considered fundamental sequences described in 1 , but the ganeralization of this notion was given by Prof. K. Kunugi. ${ }^{9)}$ According to it we shall call a sequence of neighbourhoods $\left\{v_{n}\right\}=\left\{V\left(F_{n}, \nu_{n} ; f_{n}\right)\right\} \quad$ (where $\nu_{0} \leqq \nu_{1} \leqq \cdots, F_{0} \subseteq F_{1} \subseteq \cdots$ and $f_{m} \in v_{n}$ for $m>n$ ) star sequence if it satisfies conditions beside $\left(1^{*}\right),\left(2^{*}\right)$ in 1 ,

$\left(3^{*}\right)$ we can extract from every subsequence $\left\{v_{n[\kappa]}\right\}, \kappa=0,1,2, \cdots$, $n[0]<n[1]<\cdots$, of the sequence $\left\{v_{n}\right\}$, a sequence $\left\{v_{n[\kappa(\mu)]}\right\}, \mu=0,1,2$, $\cdots, \kappa(0)<\kappa(1)<\cdots$ which satisfies conditions (1), (2), (3) in 1. For such two sequences there exists a sequence which has the relation $\prec^{10}$ to both of them.

For star sequence $\left\{v_{n}\right\}=\left\{V\left(F_{n}, \nu_{n} ; f_{n}\right)\right\}$, the limit $\lim _{n \rightarrow \infty} f_{n}(x)=f(x)$ exists almost everywhere and $\lim _{n \rightarrow \infty} \int_{c}^{a} f_{n}(x) d x$ exists for all $a \leqq c<d \leqq b$ and depends only on $f(x)$. If we call this function $f(x)$ is (E.R.)integrable, all the results, except Theorem 3 and its corollary, are valid also.

Finally we shall give an example of function which is (E.R.)integrable in the sense of this section. ${ }^{11}$

Let us consider the Cantor set in the interval $[0,1]$. For $n=0$, $1,2, \cdots$ let $I_{j}^{n}, j=1,2, \cdots, 2^{n}$ be the open interval $\left(c_{j}^{n}, d_{j}^{n}\right)$, where $c_{j}^{n}, d_{j}^{n}$ are given by $c_{j}^{n}=\sum_{\nu=0}^{n-1}\left(2 a_{\nu}\right)(1 / 3)^{n-\nu}+(1 / 3)^{n+1}, \quad d_{j}^{n}=c_{j}^{n}+(1 / 3)^{n+1}$, the numbers $a_{\nu}$ are the coefficients of $j-1$ developed with respect to base $2: j-1=\sum_{\nu=0}^{n-1} a_{\nu} 2^{\nu}$. The function in question is given as follows:

$$
\begin{aligned}
f(x) & =(-1)^{n} \frac{3}{n+1}\left(\frac{3}{2}\right)^{n} & & \text { for } \quad x \in \bigcup_{j=1}^{2^{n}} I_{j}^{n}, \\
& =0 & & \text { for } x \text { in Cantor set. }
\end{aligned}
$$

9) Cf. K. Kunugi: Sur une généralisation de l'intégrale, loc. cit. p. 20.

10) For this notation, cf. K. Kunugi: Loc. cit. p. 12.

11) This example is due to Prof. K. Kunugi: Loc. cit. p. 27. 